Elsevier

JIM 05522

\title{
A micro-perfusion chamber for single-cell fluorescence measurements
}

\author{
C. Ince ${ }^{1,2}$, R.E. Beekman ${ }^{3}$ and G. Verschragen ${ }^{4}$ \\ ${ }^{1}$ Department of Surgery, Erasmus University, Rotterdam, The Netherlands, ${ }^{2}$ Department of Medical Physics, University of Amsterdam, \\ Amsterdam, The Netherlands, ${ }^{3}$ Department of Physiology, Free University, Amsterdam, The Netherlands, and ${ }^{4}$ Department of \\ Physiology, University of Leiden, The Netherlands
}

(Received 2 October 1989, revised received 4 December 1989, accepted 6 December 1989)

A versatile closed micro-perfusion chamber designed for single-cell fluorescence measurements under maximum microscopic magnification is described. Glass coverslips with adherent cells can be attached to the top or bottom of the chamber, depending on whether an inverted or an upright microscope is used. Eight conical holes drilled in the side of the chamber serve for the insertion of plugs with attachments for perfusion, rapid injection of small amounts of reagents, temperature measurements or for heating the interior of the chamber. Materials used in the construction of the chamber are non-toxic and resistant to standard sterilization procedures. Perfusion and temperature properties of the chamber are described. Single cell fluorescence measurements are presented in human monocyte-derived macrophages in which $\mathrm{NAD}(\mathrm{P}) \mathrm{H}$ and intracellular calcium are measured.

Key words: Chamber; Perfusion; Macrophage; Intracellular calcium; NAD(P)H; Fluorescence

\section{Introduction}

Fluorescence studies in living cells are providing new insights into cell function during immune processes. The availability of viable fluorescent probes directed at intracellular ions paved the way for these studies (for a recent review see Taylor and Wang, 1989). Changes in intracellular calcium concentrations, for example, have been implicated in such diverse functions as chemotaxis (Snyderman and Goetzl, 1981), phagocytosis (Lew et al., 1985; Kruskal and Maxfield, 1987; questioned for Fc-mediated phagocytosis by McNeil et al., 1986), receptor-ligand binding (Young et al., 1984), and

Correspondence to: $\mathrm{C}$. Ince, Department of Surgery, Erasmus University, Dr. Molewaterplein 40, Rotterdam, The Netherlands. mitogenic stimulation (Tsien et al., 1982). Initially, such studies were carried out in populations of cells with the use of fluorimeters (Tsien et al., 1982; Young et al., 1984) and later, with the introduction of new techniques (Kruskal et al., 1984; Tsien et al., 1985) and probes (Grynkiewicz et al., 1985), on single cells. Such single-cell fluorescence studies revealed that changes in intracellular ionic concentrations can occur asynchronously in a population of cells (Kruskal and Maxfield, 1987) and that intracellular ionic concentrations are heterogenously distributed inside cells (Sawyer et al., 1985), thus emphasizing the value of performing single-cell measurements. In the course of such single-cell fluorescence studies in mononuclear phagocytes, we found we needed a closed multi-purpose chamber for use on a microscope. Such a chamber was needed not only to obtain maximal control of cell environment but also for 
single-cell measurements in upright microscopical systems. Based on our experience in chamber design (Ince et al., 1983a, 1985) and in single cell studies on macrophages (Ince et al., 1983b, 1988), we undertook to design, construct, and validate a versatile closed micro-perfusion chamber suited for single cell fluorescence studies.

The chamber had to provide maximum control of cell environment under maximum microscopical magnification while offering great flexibility of use and applications. To this end, the closed micro-perfusion chamber had to meet the following specifications: (1) the chamber had to be able to accommodate glass coverslips (needed for maximum magnification) for inverted as well as upright microscopes, (2) have parts made of non-toxic autoclavable materials without the use of glues and easy to assemble, (3) permit steady-state perfusion of the chamber without allowing direct streaming of perfusate over cells, (4) allow rapid injection of small amounts of reagents into cells, (5) permit temperature control inside the chamber without relying on external control of the temperature of the cell perfusate, and (6) allow insertion into the chamber of sensors for measurements of, for example, temperature, $\mathrm{pH}$, and $\mathrm{p} \mathrm{O}_{2}$. A chamber meeting these specifications is described in the present paper. Preliminary results have been presented elsewhere (Ince et al., 1988a).

\section{Materials and methods}

\section{The micro-perfusion chamber}

The mainframe of the micro-perfusion chamber (Fig. 1A) is made of a special type of hard and durable Teflon (Eriflon, Eriks, N.V., Alkmaar, The Netherlands), and has a volume of about 2 $\mathrm{ml}$. A glass coverslip with adherent cells can be attached to either the bottom or the top of the chamber, depending on whether an inverted or upright microscope is to be used. Coverslips are placed on a silicon rubber $O$ ring and are secured by a snap type of sealing between a larger $O$ ring and an outer stainless steel securing ring (Fig. $1 B$ ). Eight conical holes on the side of the chamber serve for the insertion of perfusion and other types of plug. Steady-state perfusion plugs (Fig. $2 A$ ) can be used as either inlets or outlets and their conical fixtures make them easy to remove. The perfusion plugs were tooled such that ingoing perfusate would be directed sideways. Other types of implements can be incorporated into the plugs for control or measurement of the cell environ-
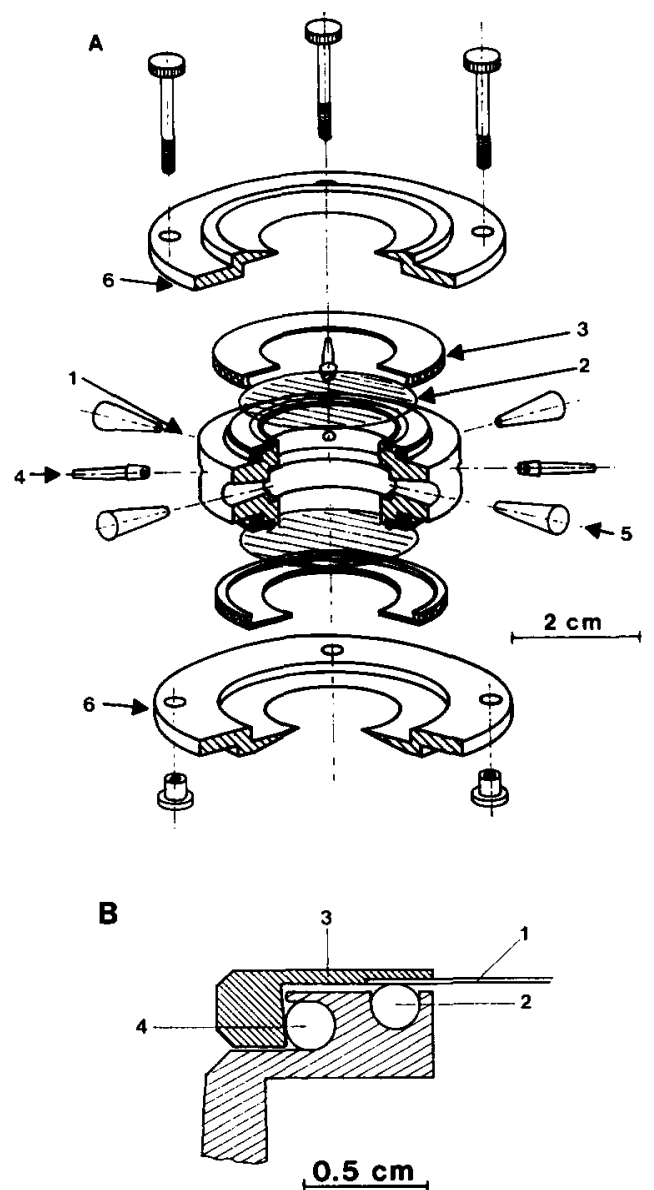

Fig. 1. Schematic representation of the components of the micro-perfusion chamber $(A)$ with a close-up of the snap-type fitting for securing glass coverslips $(B) . A$ : the mainframe of the chamber (1) is made of Eriflon, a hard durable type of Teflon. A glass coverslip with adherent cells (2) can be attached to the top or the bottom of the chamber by a metal securing ring (3). Perfusion plugs (4) or other types of plug (5) with or without probes can be inserted into conically drilled holes on the side of the chamber. Once assembled, the chamber can be placed inside a brace (6) to prevent leakage when high internal pressure occurs under high perfusion flow rates. $B$ : detail of the part of the chamber showing how the glass coverslips are secured on the mainframe. A glass coverslip (1) is placed on the inner silicon rubber $O$ ring (2) and the stainless steel securing ring (3) is snapped over the coverslip. Sealing is achieved by the pressure exerted by the larger outer $O$ ring (4) on the conically drilled stainless steel ring. 
A

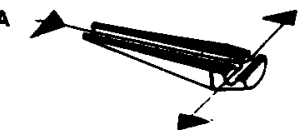

B

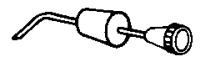

C

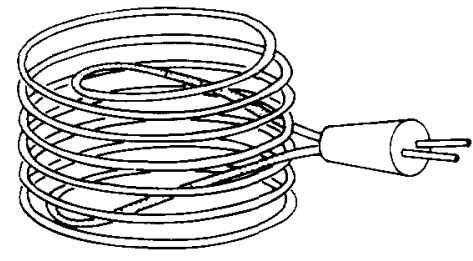

D

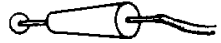

Fig. 2. Plugs for the control and measurement of environmental factors to which cells in the chamber are exposed. $A$ : cross-section of a perfusion plug in which perfusate is directed sideways through lateral slits to minimize direct streaming of perfusate toward cells. $B$ : plug permitting rapid injection of reagents into the cell invironment. $C$ : coil made of hollow strainless steel tubing carrying warm or cold fluid is used for internal temperature control for the chamber. The coil is attached to the inlet and outlets in the plug by thin silicon tubing. $D:$ a thermistor (or other sensor) can be inserted into a plug to monitor chamber conditions during measurements.

ment too, for example, a plug for incorporation of a thermistor for temperature measurements, a plug with a hooked injection needle to facilitate rapid injection of small amounts of reagent, and a plug designed for insertion into the chamber of a thin hollow coiled stainless steel tube through which a warm (or cold) fluid can be passed to obtain internal temperature control for the chamber. Once assembled, the chamber can be placed in a brace (Fig. 1A) giving greater compression to prevent leakage when high intra-chamber pressures occur during high perfusion rates.

\section{Fluorescence measurements}

Fluorescence measurements performed to determine the properties and applicability of the chamber included measurements of the kinetics of perfusate changes and single cell fluorescence measurements of $\mathrm{NAD}(\mathrm{P}) \mathrm{H}$ and intracellular calcium levels in human-monocyte derived macrophages. For studies on perfusion kinetics, use was made of an Olympus BH-2 fluorescence (up-right) microscope fitted with a dichroic mirror. UV excitation was provided by a $100 \mathrm{~W} \mathrm{Hg}$ lamp and fluorescence was measured with a conventional photomultiplier. The chamber perfusate was alternately switched between distilled water and an aqueous solution of FITC (fluorescein isothiocyanata-dextran, Pharmacia), and the photomultiplier output was recorded.

To illustrate the flexibility of the chamber, single-cell fluorescence measurements in human macrophages were carried out on an upright (NAD(P)H measurements) as well as on an inverted (intracellular calcium measurements) microscope. The NAD(P)H measurements were performed on an upright Leitz orthoplan fluorescence microscope using a $\times \mathbf{4 0}$ Zeiss neofluoar objective and illuminated with a $100 \mathrm{~W}$ mercury lamp. The microscope was fitted with filters providing 360 $\mathrm{nm}$ excitation and allowing the $460 \mathrm{~nm}$ emission associated with $\mathrm{NAD}(\mathrm{P}) \mathrm{H}$ fluorescence. Images of cells were detected by a Hamamatsu C1966-20 VIM Photonic Microscopic System (Hamamatsu Photonics, Hamamatsu City, Japan) which consisted of an ultra-sensitive video camera (capable of photon counting) coupled to an image processing system. Single-cell calcium measurements were performed with use of the fluorescent calcium indicator dye fura-2 (Grynkiewicz et al., 1985; Tsien et al., 1985) and an inverted Zeiss IM fluorescence microscope fitted with a quartz microscopic objective (Nikon $\times 40$ ). The microscope was fitted with a rotating wheel containing excitation filters and capable of 250 rotations/s, thereby providing dual excitation at wavelengths of 334 and $380 \mathrm{~nm}$. Electronic synchronization between the flywheel and the photomultiplier permitted on line monitoring of the two fluorescent signals (Kruskal et al., 1984). The emission wavelength was $510 \mathrm{~nm}$, and the intracellular calcium concentration was calculated according to Grynkiewicz et al. (1985).

\section{Cells, solutions, and reagents}

The human macrophages used for single-cell measurements were obtained by culture of periph- 
eral blood monocytes on $35 \mathrm{~mm}$ diameter glass coverslips for periods ranging between three and ten days during which the monocytes matured and became macrophages. The culture medium was Medium 199 (Microbiological Associates, MA, U.S.A.) supplemented with $10 \%$ heat-inactivated FCS (Gibco Bio-Cult, U.K). For performance of the measurements, coverslips with adherent cells were transferred to either the top or the bottom of the chamber, depending on whether upright or inverted optics were to be used. The solution in which fluorescence measurements were made was composed of $150 \mathrm{mM} \mathrm{NaCl} ; 5 \mathrm{mM} \mathrm{KCl} ; 1 \mathrm{mM}$ $\mathrm{MgCl}_{2} ; 4 \mathrm{mM} \mathrm{CaCl}$; and $10 \mathrm{mM}$ Hepes-NaOH (pH 7.2).

Toxicity of the materials used in the chamber was assessed by culturing HeLa cells in the presence of a sample of the material under study. Cells were continuously grown by trypsinization and replating of the cells at 1 week intervals. After 3 weeks of culture, the cells were counted, their morphology was examined, and the results were compared with those in the control cultures. The culture medium consisted of alpha MEM (Gibco) supplemented with $10 \%$ FCS (Gibco).

For the intracellular calcium measurements, cells were loaded with calcium indicator dye fura-2 (Molecular Probes, OR, U.S.A.) as described elsewhere (Kruskal et al., 1987). For the fura-2 measurements, $5 \mathrm{mM}$ probenecid ( $p$-(dipropylsulfamoyl)benzoic acid; Sigma Chemical Co., St. Louis, MO, U.S.A.) was added to the solution to prevent leakage of fura-2 from the cells ( $\mathrm{Di}$ Virgilio 1988). Histamine dihydrochloride was purchased from the Sigma Chemical Co.

\section{Results}

The chamber and its parts are shown in Fig. $1 A$. The snap type of fitting shown in Fig. $1 B$ and the conical fixtures for plugs make the use of glue in assembling the chamber unnecessary. The Eriflon main frame and silicon rubber $O$ rings, which come into contact with cells, are non-toxic as shown by the HeLa cell toxicity tests described in the materials and methods section. All of the components of the chamber are resistant to standard sterilization procedures (heat, steam and al- cohol). These features of the chamber and its modular structure meet the design specifications of non-toxicity, ease of assembly, flexibility of use, and resistance to sterilization.

Depending on whether use is made of an inverted or upright microscope, a coverslip with cells is placed on the bottom or the top of the chamber, the opposite opening being covered with an empty coverslip. The coverslips are attached by a snap type of fitting (Fig. $1 B$ ). This snap type of sealing was chosen instead of the bayonet fittings used previously (Ince et al., 1985) because it offered better sealing properties and greater ease of handling.

Plugs for control of or measurements in the cell environment can be inserted with a snug fit into any of the eight conical holes on the side of the chamber. Various types of plug can be used, including perfusion plugs, a plug for maintenance of internal temperature control, plugs for injecting reagents, or plugs containing sensors (Fig. 2). When cells are to be exposed to a perfusate, it is important to keep in mind that the sheer stress, exerted on cells by the movement of the fluid perfusate can elicit cellular reactions (Olesen et al., 1988). By directing the perfusate sideways through the two slits on the side of the perfusion plugs, this sheer stress can be minimized (Fig. $2 A$ ). Furthermore, the plugs can be turned in their socket to change the perfusion direction either laterally or horizontally to maximize mixing efficiency when one perfusate is exchanged for another. The kinetics of perfusate changes were followed by performing the experiment shown in Fig. $3 A$, in which use was made of two solutions, one clear and the other containing a fluorescent dye, and the effects were observed with a fluorescence microscope. The absence of a slow increase in fluorescence after switching to the solution with the fluorescent dye shows that there are no dead spaces in the chamber and that good exchange of solutions has been achieved. For rapid injection of small amounts of reagents, a plug carrying a hooked injection needle can be used (Fig. $2 B$ ). As can be seen from the trace in Fig. $3 B$, this arrangement is highly effective for rapid addition of a reagent.

Control of the temperature in the cell chamber is usually achieved by external control of the 
temperature of the cellular perfusate. For temperature control during the absence of perfusion a coil of stainless steel tubing can be connected to a plug and inserted into the chamber (Fig. $2 \mathrm{C}$ ); and a temperature-controlled solution is then pumped through this coil. The efficacy of this method of temperature control was quantified by performing the temperature jump experiment illustrated in Fig. 3C. Step-wise changes in the temperature of the perfusate flowing through the coil heated the solution bathing the cells (with zero-flow cellular perfusion) according to the time course shown in
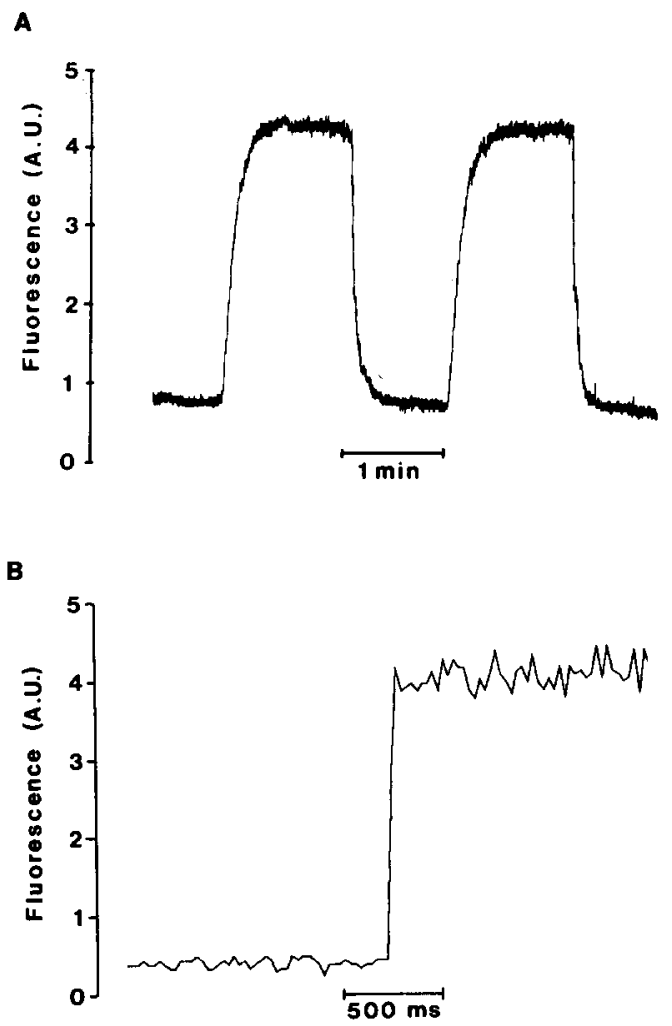

C

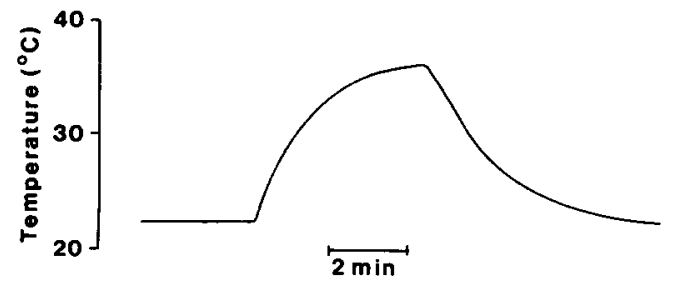

Fig. $3 C$. In this experiment the temperature was measured by a plug containing a thermistor of the type shown in Fig. $2 D$.

The symmetrical design of the chamber means that it can be used with inverted as well as upright microscopes. To illustrate the flexibility of the chamber in this respect we performed two types of single cell fluorescence measurements on human monocyte-derived macrophages, starting with the measurement of NAD(P)H autofluorescence. Besides giving a measure of cellular NADPH, this autofluorescence provides a measure of mitochondrial oxygen consumption (Chance, 1976). The level of $\mathrm{NAD}(\mathrm{P}) \mathrm{H}$ fluorescence in a single cell is exceedingly low and only recently has it become possible to detect such images without significant bleaching of the signal (Eng et al., 1989). The photograph in Fig. $4 A$ shows the intracellular distribution of $\mathrm{NAD}(\mathrm{P}) \mathrm{H}$ in a human macrophage and was taken with an ultra-sensitive video camera. An image processor enabled the quantification of the amount of cellular fluorescence by use of gray-level detection (seen in the fluorescence photograph in Fig. $4 A$ in the form of a histogram). The image shown in Fig. $4 A$ was detected within about $100 \mathrm{~ms}$, whereas a $5 \mathrm{~min}$ exposure is needed with a conventional photocamera using 400 ASA film (data not shown). For NAD(P)H fluorescence measurements, glass coverslips with adherent cells were attached to the top of the chamber.

\footnotetext{
Fig. 3. Traces showing the effects of perfusion and temperature control in the chamber. $A$ : traces obtained with perfusion plugs. During steady-state perfusion of the chamber $(1 \mathrm{ml} / \mathrm{min})$ a clear solution is exchanged for one containing a fluorescent dye. Measurement in arbitrary units (A.U.) of fluorescence of the output of a photomultiplier attached to a fluorescence microscope shows that step-wise exchange of solutions is accomplished by this method. For this example four perfusion plugs were used, two as inlet and two as outlet for the perfusate. $B$ : this trace reflects the rapid injection of a reagent with the injection plug shown in Fig. $2 B$. For our example a fluorescent dye was injected, and its time course was followed with a fluorescence microscope. $C$ : trace showing the time needed to raise the temperature of the solution bathing the cells in the chamber from room temperature to $37^{\circ} \mathrm{C}$ with the internal heating coil shown in Fig. $2 C$. First a warm and then cold solution was passed through the coil to determine the thermal time constant of the chamber. The temperature of the solution was sensed by a thermistor like the one shown in Fig.
} 

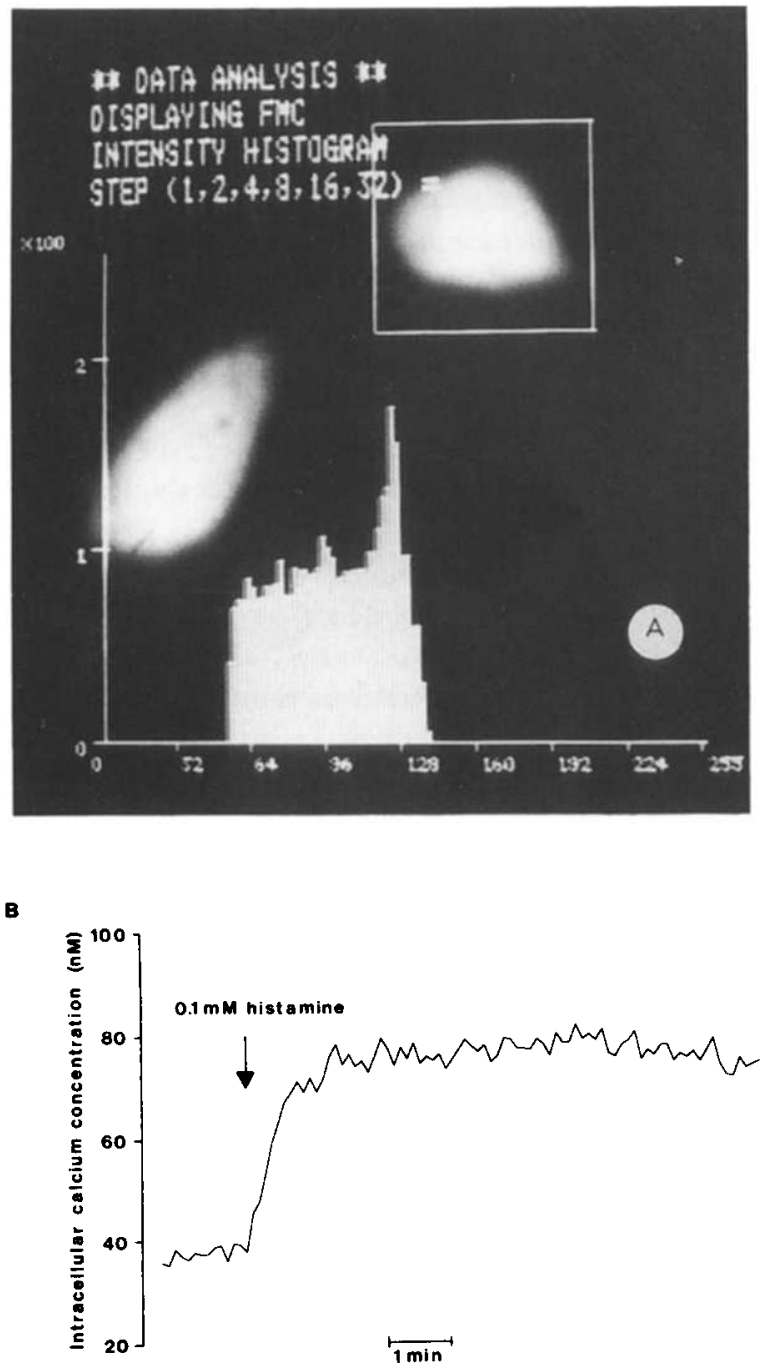

Fig. 4. Application of the micro-perfusion chamber for singlecell fluorescence microscopy in human macrophages. $A$ : $\mathrm{NAD}(\mathrm{P}) \mathrm{H}$ fluorescence in a human macrophage. Images were obtained with an upright fluorescence microscope attached to a sensitive video camera with an image intensifier. Here the coverslip with cells was attached to the top of the chamber. An image processor measured the pixel gray level distribution of the cell (seen next to the image of the cell), which was used for quantification of the intensity of the intracellular NAD(P)H fluorescence. $B$ : trace obtained during intracellular calcium measurements in a human macrophage exposed to $0.1 \mathrm{mM}$ histamine. Measurements were made with an inverted microscope. The coverslip with adherent cells was attached to the bottom of the chamber. The calcium indicator fura- 2 was used in combination with the ratio imaging method. The histamine solution was administered via the plugs used for steady-state perfusion at a flow rate of $1 \mathrm{ml} / \mathrm{min}$.
Intracellular calcium measurements have acquired an increasingly important place in the study of immune processes. The use of ratio-imaging techniques in combination with intracellular calcium indicator dyes such as fura- 2 has made it possible to measure quantitatively changes occurring in the intracellular calcium concentration in single cells. Fig. 4B shows the response of a human macrophage to exposure of $0.1 \mathrm{mM}$ histamine. Histamine was added during steady-state perfusion at a flow rate of $1 \mathrm{ml} / \mathrm{min}$, and the measurements were done in the presence of $5 \mathrm{mM}$ probenecid to avoid fura-2 leakage from cells (Di Virgilio et al., 1988). For the intracellular calcium measurements, glass coverslips with adherent cells were attached to the bottom of the chamber.

\section{Discussion}

Fluorescence measurements on single living cells makes it possible to perform both qualitative and quantitative studies on the structure and function of cells during cellular activity. Such measurements can be made with the micro-perfusion system described here. The main advantages of using a closed chamber instead of an open chamber (e.g., Ince et al., 1985) include the ability to create extreme environmental conditions (e.g., anoxia) and the possibility of using upright microscopic systems for measurements. Although several types of closed chamber have been described in the past (Sykes and Moore, 1959; Dvorak and Stotler, 1971; Berg and Block, 1984, Fantini et al., 1987), the present design provides a number of features not considered in these earlier versions. These features include: (1) the perfusion plugs designed to minimize mechanical stimulation of cells during perfusion (that sheer stress caused by flowing perfusate can stimulate cells was recently demonstrated by Olesen et al., (1988) in endothelial cells), (2) temperature control inside the chamber in the absence of thermal support created by the cellular perfusate, (3) the ability to monitor intrachamber environmental parameters (e.g., temperature, $\mathrm{pH}, \mathrm{pO}$ ), (4) ease of assembly, and (5) the measurement of chamber properties such as presented in this paper. 
The successful performance of microscopical fluorescence measurements in a closed chamber requires certain precautions. For example, trapped bubbles should not be present in the chamber, because such bubbles possess a high degree of autofluorescence and can interfere with measurements. Trapping of bubbles can be avoided: (1) if the cellular perfusate is delivered to the chamber from a pre-heated reservoir instead of using a heat exchanger in the path of perfusion to warm the perfusate leaving a reservoir at room temperature, since the latter method leads to the formation of micro-bubbles, (2) if gas tension control of the perfusate is not achieved by bubbling ( $\mathrm{pH}$ control of bicarbonate-buffered solutions can be achieved by the use of passive diffusion across a thin-walled silicon tubing (Bjornsson et al., 1988) or by placement of a drip chamber saturated with the desired gas mixture in the perfusion pathway; alternatively, of course, use can be made of buffers such as Hepes, which do not require gas tension control, and (3) if the surfaces of the interior of the chamber and the glass coverslips are cleaned with alcohol to make certain that there are no hydrophobic surfaces to trap micro-bubbles. Peristaltic pumps should be avoided (unless suitable buffer reservoirs are placed in the perfusion pathway), because they can cause cyclic variations in intrachamber pressure causing artifacts due to movement of the coverslip.

Besides single-cell fluorescence measurements the chamber also permits simultaneous light-microscopical observations with the use of such optical configurations as phase contrast, interference contrast or the recently introduced confocal scanning microscopy. Sawyer et al. (1985) used bright-field illumination in combination with fluorescence measurements to detect correlation between intracellular calcium concentration changes and chemotaxis and phagocytosis by neutrophils. Similarly, Kruskal and Maxfield (1987) combined Nomarski-differential-interference-contrast microscopy and single-cell fura- 2 measurement to detect correlation between oscillations in the intracellular calcium concentration and the frustrated phagocytosis by macrophages of glass surfaces.

The rising cost of equipment for single-cell fluorescence measurements made flexibility of application of the chamber a major design consider- ation, because this feature permits measurement in different types of microscopical configurations. To illustrate the flexibility of the chamber in this respect we performed single-cell measurements on inverted and upright microscopes. The presented $\mathrm{NAD}(\mathrm{P}) \mathrm{H}$ measurements in macrophages are, to our knowledge, the first such measurements in immune cells. In single rat myocytes, however, Eng et al. (1989) recently demonstrated that this autofluorescence originates predominantly from mitochondria. In the present study we made use of the perfusion feature of the chamber to establish the elicitation by histamine of a rise in intracellular calcium in single human macrophages. With the recent introduction of fluorescence probes directed at such parameters as membrane potential and intracellular $\mathrm{C1}^{-}, \mathrm{H}^{+}, \mathrm{K}^{+}, \mathrm{Na}^{+}$levels (for a recent review of available probes see Tsien, 1989) single-cell fluorescent measurements are expected to provide new insights into cell function. The micro-perfusion chamber presented in this paper should provide a useful tool for the performance of such single cell investigations.

\section{Acknowledgements}

The Department of Physiology of the University of Leiden, where this device is manufactured, has licensed Medical Systems Corporation, 1 Plaza Rd., Greenvale, NY 11548, U.S.A., to market the present chamber under the name 'Closed Perfusion Chamber'.

The authors are indebted to acknowledge Dr. M. Kapsenberg of the Department of Cell Biology, University of Amsterdam, for providing human macrophages and Dr. H. Tanke and Dr. P. Nederloff of the Department of Histocytochemistry of the University of Leiden for the use of the Hamamatsu Video Imaging System. They also wish to thank Mr. J. Slats for his advice on microscopic configurations.

\section{References}

Berg, H.C. and Block, S.M. (1984) A miniature flow cell designed for rapid exchange of media under high-power microscope objectives. J. Gen. Microbiol. 130, 2915. 
Bjornsson, O.G., Kobayashi, K. and Williamson, J.R. (1987) Inducers of adenylate cyclase reverse the effect of leukotriene D4 in isolated working guinea pig heart. Am. J. Physiol. 252, 1235.

Chance, B. (1976) Pyridine nucleotide as an indicator of the oxygen requirements for energy-linked functions of mitochondria. Circ. Res. 38, (suppl. 1), 131.

Di Virgilio, F., Steinberg, T.H., Swanson, J.A., Silverstein, S.C. (1988) Fura-2 secretion and sequestration in macrophages. J. Immunol. 140, 915.

Dvorak, J.A. and Stotler, W.F. (1971) A controlled-environment culture system for high resolution light microscopy. Exp. Cell Res. 68, 144.

Eng, J., Lynch, R.M. and Balaban, R.S. (1989) Nicotinamide adenine dinucleotide fluorescence spectroscopy and imaging of isolated cardiac myocytes. Biophys. J. 55, 621 .

Fantini, E., Athias, P., Courtois, M. and Grynberg, A. (1987) A simple gas-flow chamber for cultured cell electrophysiology in a controlled atmosphere. Eur. J. Physiol. 409, 632.

Grynkiewicz, G., Poenie, M. and Tsien, R.Y. (1985) A new generation of $\mathrm{Ca}^{2+}$ indicators with greatly improved fluorescence properties. J. Biol. Chem. 260, 3440.

Ince, C. and Verschragen, G. (1988) A closed micro-perfusion chamber for single cell fluorescence measurements. Eur. J. Physiol. 412, 51 (abstract).

Ince, C., Ypey, D.L. Diesselhof-Den Dulk, M.M.C., Visser, J.A.M., De Vos, A. and Van Furth, R. (1983a) Micro-CO,incubator for use on a microscope. J. Immunol. Methods 60, 269.

Ince C., Ypey, D.L., Van Furth, R. and Verveen, A.A. (1983b) Estimation of the membrane potential of cultured macrophages from the fast potential transient upon microelectrode entry. J. Cell Biol. 96, 796.

Ince, C., Van Dissel, J.T. and Diesselhoff-Den Dulk, M.M.C. (1985) A Teflon culture dish for high magnification observations and measurements from single cells. Eur. J. Physiol. 403, 240.

Ince, C., Coremans, J.M.M.C., Ypey, D.L., Leijh, P.C.J., Van Furth, R. and Verveen, A.A. (1988) Ionic channel currents accompany phagocytosis. J. Cell Biol. 106, 1873.

Kruskal, B.A. and Maxfield, F.R. (1987) Cytosolic free calcium increases before and oscillates during frustrated phagocytosis in macrophages. J. Cell Biol. 105, 2685.

Kruskal, B.A., Keith, C.H. and Maxfield, F.R. (1984) Thyrotropin releasing hormone induced changes in intracellular $\left[\mathrm{Ca}^{2+}\right]$ measured by microspectrofluorometry on individual quin2-loaded cells. J. Cell Biol. 99, 1167.

Lew, D.P., Andersson, T., Hed, J., Di Virgilio, F., Pozzan, T. and Stendahl, O. (1985) $\mathrm{Ca}^{2+}$-dependent and $\mathrm{Ca}^{2+}$-independent phagocytosis in human neutrophils. Nature 315, 509.

Olesen, S.P., Clapham, D.E., Davies, P.F. and Davies, M. (1988) Haemodynamic shear stress activates a $\mathbf{K}^{+}$current in vascular endothelial cells. Nature 331, 168.

Sawyer, D.W., Sullivan, J.A. and Mandell, G.L. (1985) Intracellular free calcium localization in neutrophils during phagocytosis. Science 230, 663.

Snyderman, R. and Goetzl, E.J. (1981) Molecular and cellular mechanism of leukocyte chemotaxis. Science $213,830$.

Sykes, J.A. and Moore, E.B. (1959) A new chamber for tissue culture. Proc. Soc. Exp. Biol. Med. 100, 125.

Taylor, D.L. and Wang, Y. (1989) Fluorescence microscopy of living cells in culture. Methods Cell Biol. 29, 30.

Tsien, R.Y. (1989) Fluorescent indicators of ion concentrations. Methods Cell Biol. 30, 127-153.

Tsien, R.Y., Pozzan, T. and Rink, T.J. (1982) T-cell mitogens cause early changes in cytoplasmic free $\mathrm{Ca}^{2+}$ and membrane potential in lymphocytes. Nature 295, 68.

Tsien, R.Y., Pozzan, T. and Rink, T.J. (1982) Calcium homeostasis in intact lymphocytes: cytoplasmic free calcium monitored with a new intracellularly trapped fluorescent indicator. J. Cell Biol. 94, 325.

Tsien, R.Y., Rink, T.J. and Poenie, M. (1985) Measurement of cytosolic free $\mathrm{Ca}^{2+}$ in individual small cells using fluorescence microscopy with dual excitation wavelengths. Cell Calcium 6, 145.

Young, J.D.-E., Ko, S.S. and Cohn, Z.A. (1984) The increase in intracellular free calcium associated with IgGy2b/y1 Fc receptor-ligand interactions: Role in phagocytosis. Proc. Natl. Acad. Sci. U.S.A. 81, 5430. 